\title{
A numerical approximation to some specific nonlinear differential equations using magnus series expansion method
}

Sure Kome ${ }^{1}$, Aytekin Eryilmaz ${ }^{1}$ and Mehmet Tarik Atay ${ }^{2}$

${ }^{1}$ Department of Mathematics, Hacibektas Veli University, Nevsehir, Turkey

${ }^{2}$ Department of Mechanical Engineering, Abdullah Gul University, Kayseri, Turkey

Received: 13 August 2015, Revised: 10 September 2015, Accepted: 14 September 2015

Published online: 30 January 2016.

\begin{abstract}
In this paper, we investigate the effect of Magnus Expansion Method which is based on Lie groups and Lie algebras on some specific nonlinear differential equations which are Liénard and Isothermal Gas Spheres Equation. Moreover, we obtain the numerical results and then we present approximate, exact values and absolute error norms in detail.
\end{abstract}

Keywords: Magnus Expansion Method, Liénard equation, isothermal gas spheres equation, geometric integration, initial value problems.

\section{Introduction}

Liénard type equations emerge in the fields of biology, physics, engineering and mechanics $[4,9,19,12]$. The second part of the 16th Hilberts problem [10] together with the Riemann conjecture are the unique two problems of the list of Hilbert.

The generalized polynomial Liénard differential equation was presented in [14] and Liénard type second order nonlinear differential equation of the form $[14,15]$

$$
y^{\prime \prime}(x)+f(y) y^{\prime}(x)+g(y)=h(x)
$$

where $f(y)$ and $g(y)$ are polynomials in the variable $y$ of degrees $n$ and $m$ respectively.

The isothermal gas sphere equation has the following form;

$$
y^{\prime \prime}(x)+\frac{2}{x} y^{\prime}(x)+e^{y(x)}=0, \quad x \geq 0
$$

subject to initial conditions

$$
y(0)=0, \quad y^{\prime}(0)=0 .
$$

More detailed information about the Eq. (2) can be found in [7]. The semi-analytical solution of the Eq. (2) was found by Wazwaz in [20], Liao in [13], Singh et al. in [18] and Ramos in [17] by using ADM, ADM, MHAM and series expansion 
respectively as

$$
y(x) \simeq-\frac{1}{6} x^{2}+\frac{1}{5.4 !} x^{4}-\frac{8}{21.6 !} x^{6}+\frac{122}{81.8 !} x^{8}-\frac{61.67}{495.10 !} x^{10} .
$$

In 1954, an exponential representation of the solution of a first order linear differential equation for a linear operator was presented by W. Magnus [16]. Magnus series have taken attention in the theory of differential equations [6] and control theory [3]. In 1999, Iserles and Norsett examined on the solution of linear differential equations in Lie groups [11]. They obtained a geometric integrator which is Magnus Expansion Method by using affordable quadrature rules. Casas and Iserles developed explicit nonlinear Magnus Expansion Method and they presented fourth order method in [5]. Atay et al. analyzed Magnus Series Expansion Method for solving nonhomogeneous stiff systems of ordinary differential equations in 2015 [1]. Also, Atay et al. presented comparative numerical solutions of stiff ordinary differential equations using Magnus series expansion method in 2015 [2].

In this paper, our aim is to show efficiency of Magnus Expansion Method on numerical solutions of some specific nonlinear differential equations which are Liénard and isothermal gas sphere equation.

\section{The Magnus expansion method for nonlinear differential equations}

In this section, we deal with the nonlinear Lie-type equation. The initial value problem of nonlinear Lie-type equaiton is considered as

$$
Y^{\prime}(t)=A(t, Y) Y \quad Y(0)=Y_{0} \in \mathfrak{G}
$$

where $\mathfrak{G}$ is a matrix Lie group, $A: \mathbb{R}^{+} \times \mathfrak{G} \rightarrow \mathfrak{g}$ and $\mathfrak{g}$ is the corresponding Lie algebra.

Firstly, we demonstrate the solution of (4) in the form,

$$
Y(t)=e^{\Omega(t)} Y_{0}
$$

We can obtain the differential equation satisifed by $\Omega$ :

$$
\Omega^{\prime}=d \exp _{\Omega}^{-1}(A(t, Y))=d \exp _{\Omega}^{-1}\left(A\left(t, e^{\Omega(t)} Y_{0}\right)\right), \quad \Omega(0)=O .
$$

where,

$$
d \exp _{\Omega}^{-1}(C)=\sum_{k=0}^{\infty} \frac{B_{k}}{k !} a d_{\Omega}^{k} C
$$

$\left\{B_{k}\right\}_{k \in Z^{+}}$are the Bernoulli numbers $\left(B_{0}=1, B_{1}=-\frac{1}{2}, B_{2}=\frac{1}{6}, B_{3}=0, \ldots\right)$. Note that, all odd-indexed Bernoulli numbers except for $B_{1}$ are zero and $a d^{m}$ is adjoint representation which defined as follows:

$$
a d_{\Omega}^{0} A=A, \quad a d_{\Omega}^{m+1} A=\left[\Omega, a d_{\Omega}^{m} A\right], \quad m \geq 0 .
$$

The nonlinear Magnus series for $\Omega$ can be obtained by Picard's iteration formula,

$$
\Omega^{[0]}(t) \equiv O
$$




$$
\begin{aligned}
\Omega^{[m+1]}(t) & =\int_{0}^{t} d \exp _{\Omega^{[m]}(s)}^{-1} A\left(s, e^{\Omega^{[m]}(s)} Y_{0}\right) d s \\
& =\int_{0}^{t} \sum_{k=0}^{\infty} \frac{B_{k}}{k !} a d_{\Omega^{[m]}(s)}^{k} A\left(s, e^{\Omega^{[m]}(s)} Y_{0}\right) d s, \quad m \geq 0 .
\end{aligned}
$$

The power series of $\Omega^{[k]}(t)$ and $\Omega^{[k+1]}(t)$ differ in terms of order $O\left(t^{m+1}\right)$. Then we can omit all terms of $\Omega^{[k]}(t)$ with the order greater than $O\left(t^{m}\right)$. This necessitates careful analysis of each term in the expansion. If $\Omega^{[0]}=O$ and if we take $m=0$ for the first step, then

$$
\begin{gathered}
\Omega^{[1]}=\int_{0}^{t} \sum_{k=0}^{\infty} \frac{B_{k}}{k !} a d_{\Omega^{[0]} \equiv O}^{k} A\left(s, e^{\Omega^{[0]} \equiv O} Y_{0}\right) d s \\
\Omega^{[1]}=\int_{0}^{t}\left(\frac{B_{0}}{0 !} a d_{O}^{0} A\left(s, Y_{0}\right)+\frac{B_{1}}{1 !} a d_{O}^{1} A\left(s, Y_{0}\right)+\frac{B_{2}}{2 !} a d_{O}^{2} A\left(s, Y_{0}\right)+\ldots\right) d s
\end{gathered}
$$

Here, except first terms in the integral, all the terms are zero because of the zero multiplication of the adjoint representation. In that case, $\Omega^{[1]}$ is equal to only to the first term in the integral, for this reason

$$
\Omega^{[1]}=\int_{0}^{t} A\left(s, Y_{0}\right) d s=\Omega(t)+O\left(t^{2}\right)
$$

When $\Omega^{[3]}(t)$ is computed, it turns out that $\Omega^{[3]}(t)$ reproduces correctly the expression of $\Omega^{[2]}(t)$ up to $O\left(t^{2}\right)$. So, it can be truncated $d \exp ^{-1}$ at $k=0$ term and take

$$
\Omega^{[2]}(t)=\int_{0}^{t} A\left(s, e^{\Omega^{[1]}(s)} Y_{0}\right) d s
$$

Consequently, Casas and Iserles [5] showed that, if it can be truncated $d \exp ^{-1}$ at $k=m-2$ term, it is obtained explicit approximations as follows:

$$
\begin{gathered}
\Omega^{[1]}(t)=\int_{0}^{t} A\left(s, Y_{0}\right) d s, \\
\Omega^{[m]}(t)=\sum_{k=0}^{m-2} \frac{B_{k}}{k !} \int_{0}^{t} a d_{\Omega^{[m-1]}(s)}^{k} A\left(s, e^{\Omega^{[m-1]}(s)} Y_{0}\right) d s, \quad m \geq 2 .
\end{gathered}
$$

Now,it is time to compute the values of the integrals by using numerical quadrature formulas. Casas and Iserles created numerical integrator based on the nonlinear Magnus Expansion in [5] as follows.

\subsection{Nonlinear Magnus Expansion Method with order three}

$$
\left.\Omega^{[3]}(t)=\int_{0}^{t}\left(A_{2}(s)-\frac{1}{2}\left[\Omega^{[2]}(s), A_{2}(s)\right]\right)\right) d s
$$

where $A_{2}(s) \equiv A\left(s, e^{\Omega^{[2]}(s)} Y_{0}\right)$. If we use Simpson's rule, we have

$$
\Omega^{[3]}(h)=\frac{h}{6}\left(A\left(0, Y_{0}\right)+4 A_{2}\left(\frac{h}{2}\right)+A_{2}(h)\right)-\frac{h}{3}\left[\Omega^{[2]}\left(\frac{h}{2}\right), A_{2}\left(\frac{h}{2}\right)\right]-\frac{h}{12}\left[\Omega^{[2]}(h), A_{2}\left(\frac{h}{2}\right)\right]+O\left(h^{4}\right) .
$$




\subsection{Nonlinear Magnus Expansion Method with order four}

$$
\Omega^{[4]}(t)=\sum_{k=0}^{2} \frac{B_{k}}{k !} \int_{0}^{t} a d_{\Omega^{[3]}(s)}^{k} A_{3}(s) d s
$$

where $A_{3}(s) \equiv A\left(s, e^{\Omega^{[3]}(s)} Y_{0}\right)$. If we use Simpson's rule, we have

$$
\begin{aligned}
\Omega^{[4]}(h) & =\frac{h}{6} A\left(0, Y_{0}\right)+\frac{h}{6} 4 A_{3}\left(\frac{h}{2}\right)-\frac{h}{3}\left[\Omega^{[3]}\left(\frac{h}{2}\right), A_{3}\left(\frac{h}{2}\right)\right]+\frac{h}{18}\left[\Omega^{[3]}\left(\frac{h}{2}\right),\left[\Omega^{[3]}\left(\frac{h}{2}\right), A_{3}\left(\frac{h}{2}\right)\right]\right]+\frac{h}{6} A_{3}(h) \\
& -\frac{h}{12}\left[\Omega^{[3]}(h), A_{3}(h)\right]+\frac{h}{72}\left[\Omega^{[3]}(h),\left[\Omega^{[3]}(h), A_{3}(h)\right]\right] .
\end{aligned}
$$

\section{Numerical examples}

In this section, we applied fourth-order Magnus Expansion Method for nonlinear equations(NMEM4). Then, we illustrated approximate, exact solutions and absolute errors of each problems with figures in detail. Later, we presented the absolute errors with detailed tables.

Example 1. Consider the following Liénard Equation

$$
\left\{\begin{array}{l}
y^{\prime \prime}(x)+y(x) y^{\prime}(x)+y(x)+y^{2}(x)=\cos ^{2}(x)-\sin (x) \cos (x), \\
y(0)=1, \quad y^{\prime}(0)=0
\end{array}\right.
$$

whose analytical solution is the function $y(x)=\cos (x)$ [8].

Table 1: Numerical values of approximate, exact and maximum error results obtained from NMEM4 for ,Eq.(16) with $t \in[0,0.5], h=0.001$.

\begin{tabular}{lccc}
\hline$h$ & NMEM4 & Exact & Absolute error \\
\hline 0 & 1 & 1 & 0 \\
0.1 & 0.994860075659013 & 0.9950041652780258 & $1.4409 \times 10^{-4}$ \\
0.2 & 0.9789118152487274 & 0.9800665778412416 & $1.15476 \times 10^{-3}$ \\
0.3 & 0.9514346422581428 & 0.955336489125606 & $3.90185 \times 10^{-3}$ \\
0.4 & 0.9118477951509781 & 0.9210609940028851 & $9.2132 \times 10^{-3}$ \\
0.5 & 0.8597626237202116 & 0.8775825618903728 & $1.78199 \times 10^{-2}$ \\
\hline
\end{tabular}

Example 2. Consider the isothermal gas sphere equation which is given in Eq.(2),

$$
\left\{\begin{array}{l}
y^{\prime \prime}(x)+\frac{2}{x} y^{\prime}(x)+e^{y(x)}=0, \quad x \geq 0 \\
y(0)=0, \quad y^{\prime}(0)=0
\end{array}\right.
$$


Table 2: Numerical values of approximate, exact and maximum error results obtained from NMEM4 for Eq.(17) with $t \in[0,1]$, and $h=0.001$.

\begin{tabular}{lccc}
\hline$h$ & NMEM4 & Exact & Absolute error \\
\hline 0 & 1 & 1 & 0 \\
0.2 & 0.9987728129004231 & 0.9933687175402979 & $5.4041 \times 10^{-3}$ \\
0.4 & 0.9911258298643896 & 0.9738914041322059 & $1.72344 \times 10^{-2}$ \\
0.6 & 0.9727490268397473 & 0.9427594942946572 & $2.99895 \times 10^{-2}$ \\
0.8 & 0.9411702372084342 & 0.9017788097837728 & $3.93914 \times 10^{-2}$ \\
1 & 0.8955041241743008 & 0.8531436383703529 & $4.23605 \times 10^{-2}$ \\
\hline
\end{tabular}

\section{Conclusion}

In this paper, we have presented the numerical solutions of Liénard and Isothermal gas sphere equations by using Magnus series expansion method. As it can be seen in tables, Magnus series expansion method is a reliable method for the numerical solutions of nonlinear ordinary differential equations but not so powerful. Because, Magnus expansion is an explicit method.

\section{References}

[1] Atay M.T.; Eryilmaz A.; Kome S., Magnus Series Expansion Method for solving nonhomogeneous stiff systems of ordinary differential equations, Kuwait Journal of Sciences, (Accepted).

[2] Atay M.T.; Eryilmaz A.; Kome S.; Kome C.; Piipponen S., Comparative numerical solutions of stiff ordinary differential equations using Magnus series expansion method, New Trends in Mathematical Sciences 3(1):35-45, (2015).

[3] Brocket R. W., Volterra series and geometric control theory, Automatica 12:167-176, (1976).

[4] Burton T. A., Stability and periodic solutions of ordinary and functional differential equations, AcademicPress, Orlando, (1985).

[5] Casas F.; Iserles A., Explicit Magnus expansions for nonlinear equations,J. Phys. A: Math. Gen. 39:5445-5461, (2006).

[6] Chen K. T., Integration of paths, geometric invariants, and a generalized Baker-Hausdorff formula,Annals Math. 67:164-178, (1957).

[7] Davis H. T., Introduction to nonlinear differential and integral equations, Dover, New York, (1962).

[8] Gámez D.; Garralda Guillem A. I.; Ruiz Gálan M., Nonlinear initial-value problems and Schauder bases, Nonlinear Analysis 63:97-105, (2005).

[9] Hale J. K., Theory of functional differential equations, Springer-Verlag, New York, (1977).

[10] Hilbert D., Mathematische probleme (lecture), Nachr. Ges. Wiss. Gottingen Math.-Phys. K1. 253-297, (1900).

[11] Iserles A.; Norsett S. P., On the solution of linear differential equations in lie groups, Phil. Trans. R. Soc. A 357:983-1019, (1999).

[12] Kuang Y., Delay differential equations: with applications in population dynamics, AcademicPress, New York, (1993).

[13] Liao S., A new analytic algorithm of Lane Emden type equations, Appl. Math. Comput. 142:1-16, (2003).

[14] Liénard A., Étude des oscillations entreténues, Revue générale de lélectricité 23:901-912, (1928).

[15] Liénard A., Étude des oscillations entreténues, Revue générale de lélectricité 23:946-954, (1928).

[16] Magnus W., On the exponential solution of differential equations for a linear operator, Comm. Pure and Appl. Math. 7:639-673, (1954).

[17] Ramos J. I., Series approach to the Lane Emden equation and comparison with the homotopy perturbation method,Chaos Solitons Fractals 28:400-408, (2008).

[18] Singh O. P.; Pandey R. K.; Singh V. K., An analytic algorithm of Lane Emden type equations arising in astrophysics using modified homotopy analysis method, Comput. Phys. Commun. 180:1116-1124, (2009).

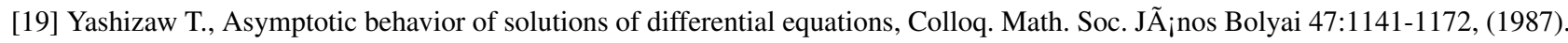

[20] Wazwaz A., A new algorithm for solving differential equations of Lane Emden type, Appl. Math. Comput. 118:287-310, (2001). 